\title{
THE EFFECT OF INCLINATION ANGLE ON CRITICAL HEAT FLUX IN A LOCALLY HEATED LIQUID FILM MOVING UNDER THE ACTION OF GAS FLOW IN A MINI-CHANNEL
}

\author{
Egor M. Tkachenko ${ }^{1,2, *}$, and Dmitry V. Zaitsev ${ }^{1}$ \\ ${ }^{1}$ Kutateladze Institute of Thermophysics SB RAS, 630090 Novosibirsk, Russia \\ ${ }^{2}$ Novosibirsk State University, 630090 Novosibirsk, Russia
}

\begin{abstract}
Intensively evaporating liquid films moving under the action of the cocurrent gas flow in a microchannel are promising for the use in modern cooling systems of semiconductor devices with high local heat release. This work has studied the dependence of the critical heat flux on the inclination angle of the channel. It has been found that the inclination angle in the plane parallel to the flow has no significant effect on the critical heat flux. Whereas the inclination angle in the plane perpendicular to the flow, on the contrary, significantly changes the value of the critical heat flux. However, for a given flow rate of fluid there is a threshold gas velocity at which the critical heat flux does not differ from the case of zero inclination of the channel. Thus, it can be concluded that the cooling system based on shear-driven liquid films can be potentially used when direction of the gravity changes.
\end{abstract}

\section{Introduction}

The development of modern microelectronic equipment requires efficient cooling systems because of the need to remove high heat fluxes, up to $1 \mathrm{~kW} / \mathrm{cm}^{2}$ from local areas of the processor [1]. Thin and ultrathin $(<10 \mu \mathrm{m})$ liquid films moving under the action of a forced gas or vapor flow in the channel are promising for the use in thermal control systems of modern semiconductor devices [2]. Works [3-5] are devoted to analytical and numerical study of hydrodynamics and heat and mass transfer during joint motion of intensively evaporating liquid film and gas flow in microchannels. Authors of experimental works [68] established the basic laws of the flow and crisis phenomena in the liquid film, moving under the action of the gas flow in the horizontal channel with $1 \times 1 \mathrm{~cm}$ heater (simulating an electronic device). Thermal control of electronic devices in transportation, aircrafts and space vehicles requires the analysis of the influence of the gravity direction on the cooling system efficiency. This paper presents experimental data on the critical heat flux for different inclination angles of the working area to the horizon.

\footnotetext{
* Corresponding author: egor tkachenko@mail.ru
} 


\section{Experimental setup}

Figure 1 shows the scheme of the work area. Its main part is a thin plate of stainless steel with imbedded copper rod. The surface of the rod has a shape of a square head of $1 \times 1 \mathrm{~cm}$ and imitates a computer chip. The rod is heated using a spiral wound around its lower part. This design of the heater provides a constant temperature on the rod surface, $\mathrm{T}=$ const (as confirmed by thermocouple measurements). The work area is covered with a transparent cover made of optical glass to form a flat channel. The height of the channel is $1.5 \mathrm{~mm}$. Gas is fed into the work area with a compressor, passes through it and is vented to atmosphere at the end of the work area. The gas flow rate is measured with a digital flow meter Bronkhorst with an accuracy of about $1 \%$.

The liquid is supplied from the thermostat, enters the channel through the liquid nozzle and moves under the action of friction from the gas over the stainless steel plate in the form of a film. Fluid then accumulates in the bottom of the work area and returns to the thermostat. The fluid flow rate is measured by the float-type flow meter with an accuracy of $1.5 \%$. The distance from the gas inlet to the liquid nozzle is $57 \mathrm{~mm}$, and from the liquid nozzle to the heater, is $32 \mathrm{~mm}$. This ensures the stability of gas and liquid flows by the time they reach the heater. The channel width is $30 \mathrm{~mm}$. Several thermocouples are embedded in the stainless steel plate and in the copper rod, as shown in fig. 1. This allows determining the working temperature of the surface. The surface temperature of the heater is calculated based on the depth at which the thermocouples are installed $(2 \mathrm{~mm})$. All thermocouples are calibrated individually with an accuracy of $0.1^{\circ} \mathrm{C}$.

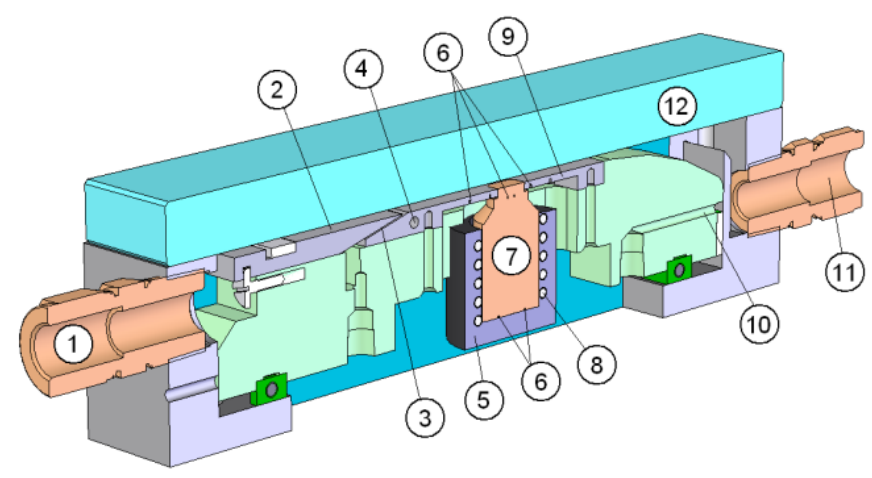

Fig. 1. The scheme of the work area: $1-$ gas inlet; $2-$ channel; $3-$ liquid nozzle; $4-$ thermal stabilizer; 5 - insulation; 6 - thermocouples; 7 - copper rod, 8 - nichrome spiral; 9 - stainless steel plate; 10 - liquid outlet; 11 - gas outlet; 12 - glass cover.

Heat flux is determined according to the electric power of the heating spiral. The thermal conductivity of copper is $400 \mathrm{~W} / \mathrm{mK}$, which is almost 30 times higher than the one of stainless steel $(15 \mathrm{~W} / \mathrm{mK})$. This provides a reasonable heat spread from the heater to the stainless steel plate. According to evaluation based on the measurements of thermocouples embedded in the stainless steel substrate, the heat spreading is of $10-15 \%$ at $\mathrm{q}>250 \mathrm{~W} / \mathrm{cm}^{2}$, about $20 \%$ at $\mathrm{q}=100 \mathrm{~W} / \mathrm{cm}^{2}$ and up to $40 \%$ for small heat fluxes. To minimize the heat spreading from the heating spiral to the atmosphere, the spiral was wrapped with a layer of thermal insulation material (mineral wool, see 5 in fig.1).

Distilled water with initial temperature of $24^{\circ} \mathrm{C}$ is used as the working fluid. Air with a temperature of $24-27^{\circ} \mathrm{C}$ and a relative humidity of $15-30 \%$ is used as working gas. Atmospheric pressure during the experiments varies from 98500 to $101600 \mathrm{~Pa}$. The experiments were carried out in stationary conditions. The gas velocity (volumetric gas 
flow rate divided by the area of the channel cross section) $U_{\mathrm{Sg}}$ varies from 0.1 to $34 \mathrm{~m} / \mathrm{s}$. Maximum pressure drop along the channel is observed at the maximum gas flow rate and estimated to be less than $500 \mathrm{~Pa}$.

\section{Results}

In this work we investigated how the inclination angle in the flow symmetry plane (the section plane in fig. 1), $\Theta$, affects the flow, breakup and critical heat flux. The inclination angle changes with the step of $45^{\circ}$ from 0 (horizontal channel) to $315^{\circ}\left(-45^{\circ}\right)$. The slope of the channel in this plane does not break the symmetry of the flow. Figure 2 shows the critical heat flux $q_{\text {cr }}$ for different inclination angles, $\Theta$, at Reynolds number of liquid $\operatorname{Re}_{1}=14$. It is seen that the critical heat flux weakly depends on the inclination angle, except for the case $\Theta=-90^{\circ}$, where two points fall out of the generalization. This is probably due to the fact that these two points lie in the region of the channel flooding, where the heater is periodically washed with liquid, delaying the onset of the heat transfer crisis.

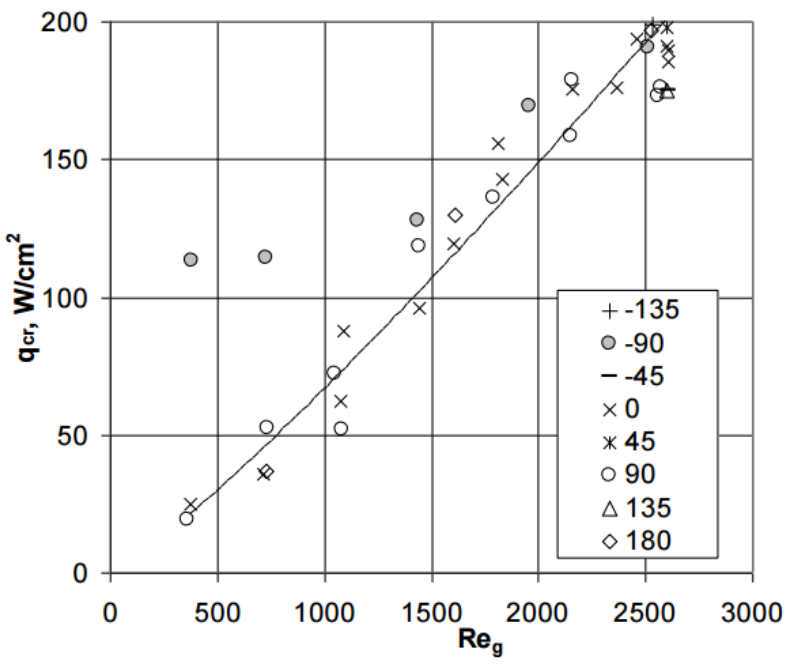

Fig. 2. The influence of the Reynolds number of the gas on critical heat flux for different $\Theta$ (shown in the legend), $\operatorname{Re}_{\mathrm{l}}=14$.

We have studied only one case of channel inclination along the plane perpendicular to the flow: $\Omega=90^{\circ}$. However, due to the symmetry of the channel, the case $\Omega=-90^{\circ}$ does not differ from the case $\Omega=90^{\circ}$. In addition, the case $\Omega=180^{\circ}$ is the same as the case $\Theta=$ $180^{\circ}$. The channel slope in this plane breaks the flow symmetry. Figure 3 shows the critical heat flux $\mathrm{q}_{\mathrm{cr}}$ for $\Omega=90^{\circ}$, at $\mathrm{Re}_{1}=30$ and $\mathrm{Re}_{1}=46$. Lines represent values $\mathrm{q}_{\mathrm{cr}}$ corresponding to the inclination angles $\Omega=0$ and $\Theta=0$. It is seen that the inclination angle along the plane perpendicular to the flow has a significant influence on the critical heat flux (flow symmetry is broken). However, for each $\mathrm{Re}_{1}$ there exists a critical value of gas velocity, starting from which the critical heat flux almost coincides with the case $\Omega=0$ and $\Theta=0$. 


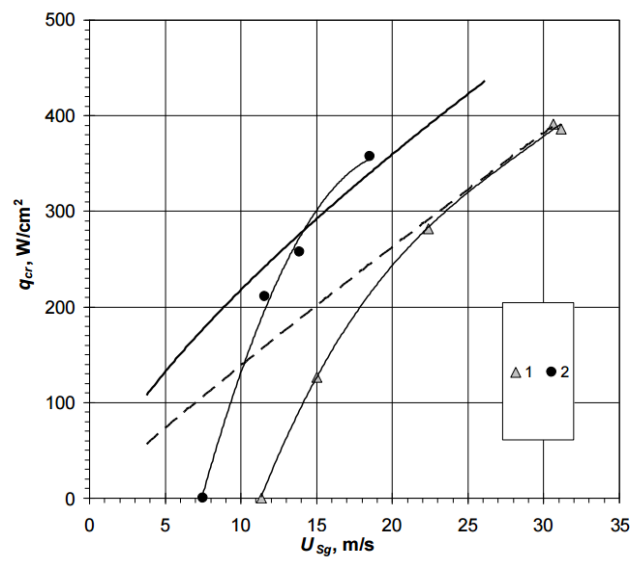

Fig. 3. Critical heat flux $q_{c r}$ for $\Omega=90^{\circ}, 1-\operatorname{Re}_{1}=30$, and $2-\operatorname{Re}_{1}=46$. Straight lines represent the values of $\mathrm{q}_{\mathrm{cr}}$, corresponding to the angles $\Omega=0$ and $\Theta=0$.

\section{Conclusions}

Thus, it can be concluded that the flow, the breakup and the critical heat flux of sheardriven liquid films weakly depend on the inclination angle in the channel symmetry plane (when the symmetry of the flow is preserved). And, on the contrary, the inclination angle in the plane perpendicular to the flow (when the flow symmetry is broken) has a significant impact on the patterns of the film flow and the crisis phenomena. However, for each value of the liquid flow rate there is a critical value of the gas velocity, starting from which the flow, the breakup and the critical heat flux have practically no dependence on the inclination angle in both planes. This shows that the cooling system, based on the evaporating liquid film moving in the channel under the action of gas flow, can be potentially used in transportation, aircraft and spacecraft, when the direction of gravity changes.

\section{Acknowledgments}

This work was supported by the Ministry of Education and Science of Russia (Agreement No. 14.604.21.0053, project identifier RFMEFI60414X0053).

\section{References}

1. A. Bar-Cohen, P. Wang, ASME. J. Heat Transf. 134 (2012)

2. O.A. Kabov, Yu. V. Lyulin, I.V. Marchuk, and D.V. Zaitsev, Int. J. Heat Fl. Flow 28 (2007)

3. E.Ya. Gatapova, O.A. Kabov, Int. J. Heat Mass Transf. 51 (2008)

4. Y. O. Kabova, Tech. Ph. Let. 39 (2013)

5. Y. Kabova, O. Kabov, T. Gambaryan-Roisman, P. Stephan, V.V. Kuznetsov, Int. J. Heat Mass Transf. 68 (2014)

6. D.V. Zaitsev, D.A. Rodionov, O.A. Kabov, Tech. Ph. Let. 35 (2009)

7. O.A. Kabov, and D.V. Zaitsev, Multiph. Sci. Tech. 21 (2009)

8. O.A. Kabov, D.V. Zaitsev, V.V. Cheverda, and A. Bar-Cohen, Exp. Therm Fluid Sci. 35 (2011) 